\title{
Aproximaciones teóricas alrededor del diseño y uso de modelos de análisis tridimensional de movimiento dentro del marco de la rehabilitación funcional
}

\author{
Jorge Enrique Correa Bautista*
}

\begin{abstract}
Resumen
El propósito de este artículo es realizar una revisión teórica alrededor del uso de modelos de análisis de movimiento tridimensionales utilizados para describir la cinemática y la cinética del gesto de movimiento corporal humano, como marco referencial para fomentar su utilización en el campo de la rehabilitación funcional.
\end{abstract}

Palabras clave: biomecánica, cinemática, análisis de movimiento, rehabilitación

\section{Introducción}

"Actividades humanas como el deporte, el trabajo, evidencian una inteligencia cinestésica-corporal evolucionada caracterizada por la habilidad de emplear el cuerpo en formas diferenciadas y complejas para propósitos expresivos orientados a la intersubjetividad del sujeto como ser social" (Mena Bejarano, 1998).

Los profesionales de las ciencias de la salud que tienen como interés mejorar la capacidad del movimiento humano, ya sea potencializándolo o mejorando su disfunción deben apoyarse en procesos de medición y de evaluación cinemática y cinética.

En este sentido, la biomecánica toma relevancia ya que es la ciencia encargada de analizar el movimiento corporal humano dentro de una actividad particular, empleándose como método de evaluación efectivo del movimiento corporal hu- mano. El estudio biomecánico se centra en analizar las variables que causan y modifican el movimiento, o la descripción de las características de éste.

De esta manera, las propiedades biomecánicas del movimiento y sus alteraciones se pueden estudiar por medio de métodos observacionales y descriptivos, los cuales permiten obtener un análisis cuantitativo o cualitativo de éste. El análisis cuantitativo consiste en una descripción del gesto motor en general o de sus partes en términos cuantitativos, a través del empleo de instrumentos o equipos especializados, con el fin de deter-

* Fisioterapeuta. Especialista en Docencia universitaria y Gerencia de las organizaciones de Salud. Candidato a maestría en Fisiología, Universidad Nacional. Catedrático de la Escuela Colombiana de Rehabilitación. Director de la Línea de Investigación en Ejercicio Físico y Desarrollo Humano y profesor de la Facultad de Rehabilitación y Desarrollo Humano de la Universidad del Rosario. jecorrea@urosario.edu.co 
minar las variables cinemáticas y cinéticas del movimiento. Este tipo de análisis ayuda a evitar la subjetividad en los procesos de evaluación, permitiendo que el proceso tenga mayor grado de confiabilidad y validez. Por otro lado, el análisis cualitativo describe también la calidad del movimiento sin el empleo de mediciones ni cálculos aritméticos. Este tipo de análisis identifica los componentes involucrados en el mismo y luego los evalúa mediante la comparación y la formulación de juicios.

Indagar en el diseño de instrumentos especializados de evaluación de movimiento que sean cada vez más precisos toma relevancia en la medida en que el clínico se apoya en el uso de grabaciones en video, la digitalización de imágenes por computador, los kinegramas, el acelerómetro, la electrogoniometría, junto con la utilización de programas informáticos especializados "software", los cuales permiten realizar un análisis biomecánico detallado de la ejecución del movimiento corporal humano en un determinado contexto.

Es aquí, donde la Fisioterapia, como disciplina de la salud y la rehabilitación, debe emplear dichas herramientas de precisión encaminadas al mejoramiento de la comprensión del gesto de movimiento, permitiendo así su utilización en diferentes espacios, en los cuales se puedan identificar las alteraciones de movimiento, el correcto diseño de aditamentos como ortesis y prótesis, y el análisis ergonómico, entre otros.

En el campo de la rehabilitación funcional, la aplicación de nuevas herramientas para el análisis del movimiento ha permitido una mejora en los procedimientos de evaluación y diagnóstico elevando la efectividad en la intervención e identificando deficiencias cinéticas de procesos patológicos crónicos del aparato osteomuscular que pasan desapercibidos con los métodos de evaluación tradicional.

\section{Primeras evidencias en el uso de modelos de análisis de movimiento}

Los primeros avances en el uso de herramientas de informática aplicada en la clínica se dieron gracias a las técnicas que permitían la reproducción de imágenes, entre ellas, se puede mencionar la fotografía, la cual realiza un registro de imágenes bidimensionales, siguiendo la trayectoria del movimiento en el espacio bidimensional, con el fin de analizar una secuencia de patrones motores.

Otra técnica por imágenes, precursora en el uso de la informática en el análisis de movimiento ha sido la cinematografía. Este tipo de reproducción de imágenes depende de la velocidad del movimiento y la distancia en que se realiza. Una de las dificultades de esta técnica es que no permite almacenar la información, evitando así la sincronización de las películas, lo que no facilita el registro del movimiento. Para analizar cuantitativamente una destreza motora mediante la videocinta es necesario crear estrategias de sincronización y almacenamiento de imágenes. Blievernicht (1967) presentó el diseño de un dispositivo rotatorio en forma de cono que permitió la sincronización de tres cámaras en tres diferentes planos. Este dispositivo generó el desarrollo de sistemas de análisis de movimiento automáticos.

Posteriormente, se creó un sistema que permitió realizar despliegues virtuales a escala en conexión con un ordenador de movimientos corporales. Estos sistemas de análisis fueron creados con el fin de estudiar de manera detallada la marcha de las personas sanas o con alguna alteración durante su ejecución.

Posteriormente, los desarrollos investigativos se centraron en la identificación de las características de la marcha, su sincronización en función de la velocidad y el período de tiempo de cada 
una de sus fases, como bien lo demostró en sus estudios Milner y Quanbury (2003) quienes investigaron la marcha de sujetos hemipléjicos utilizando únicamente interruptores de pie y la medición de la velocidad de la marcha, donde se observó que la velocidad de la marcha y la simetría de las duraciones de apoyo de una sola extremidad están estrechamente relacionadas con el grado de hemiplejía. Luego, se desarrollaron goniómetros electrónicos y acelerómetros para el registro continuo de los ángulos articulares en un movimiento, combinando fotoceldas, rayos de luces y cronómetros para medir la velocidad del movimiento.

Aunque la aplicación de estos métodos ha generado toda una revolución tecnológica respecto al análisis cinemático del movimiento, es importante tener en cuenta la determinación de la fiabilidad y validez de las mediciones realizadas. Al retomar estos conceptos se hace referencia a la exactitud y precisión de las mediciones con instrumentos diseñados, los cuales al final de cada estudio deben generar resultados precisos.

\section{Modelos tridimensionales y su aplicación}

Gracias a los estudios anteriormente comentados, se han creado una serie de sistemas de análisis de movimiento, con base en la animación tridimensional como se evidenció en el XIV Congreso Internacional de Ingeniería Gráfica, en Santander, España (julio de 2002) en colaboración con el Departamento de Ingeniería Mecánica de la Universidad de Di Roma Tor Vergara, Italia, quienes presentaron un maniquí 3-D (programa Working Model) del cuerpo humano, personalizado con las medidas de los datos antropométricos del sujeto, junto con el desarrollo de sistemas específicos de animación como el APAS (Performance Analysis System) diseñado para el análisis del movimiento mediante telecámaras de grabación simultánea. Dicho programa registra desplazamientos y aceleraciones con base en mar- cadores reflectivos dispuestos sobre el cuerpo del sujeto. Luego, el modelo fue implementado para obtener datos de la fuerza y cinética del movimiento del sujeto estudiado (Pezzoti, Biancolini, 2000).

Su ámbito de aplicación ha sido tradicionalmente el diagnóstico y control de las alteraciones del sistema locomotor con valoración y medición de los trastornos y de las secuelas de una forma precisa. Se utilizan sistemas sofisticados de análisis tridimensional del movimiento, electromiografía dinámica, aplicados a la columna vertebral (cervical, dorsal, lumbar), extremidad superior (mano, muñeca, codo, hombro), extremidad inferior (pie, tobillo, rodilla, cadera, y todo el sistema músculo esquelético.

Este análisis del movimiento consiste en la filmación tridimensional del movimiento humano con sistemas de video de alta resolución, digitalización de las imágenes por ordenador y su proceso para la medición de todos los parámetros dinámicos del movimiento del modelo biomecánico. Su utilidad se centra en la medición de la capacidad de movimiento articular y su valoración prequirúrgica y correctiva. (Figura 1)

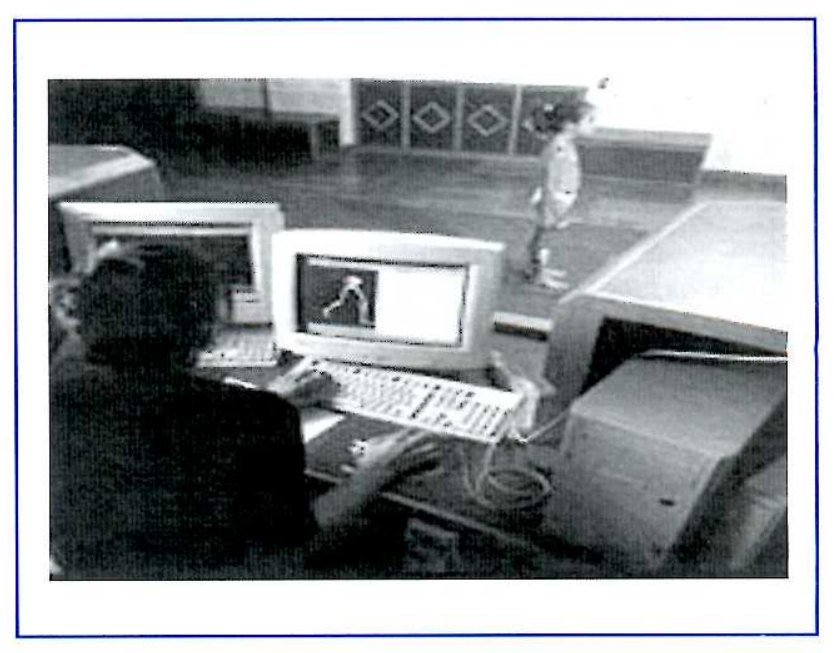

Figura 1. Laboratorio de análisis de movimiento. Instituto CIREC (Centro Integral de Rehabilitación de Colombia) 
Asimismo, se ha creado un sistema de captura de movimiento, el cual permite registrar y visualizar en tiempo real el movimiento de las articulaciones del cuerpo humano. La principal ventaja de este sistema es que puede operar completamente, aún utilizando un equipo PC de bajo costo; otras ventajas importantes son el bajo costo de hardware del sistema y de los sensores, que no requiere de ambientes controlados y el tiempo requerido para su calibración es mínimo.

El hardware de este sistema está formado básicamente por un microcontrolador, un multiplexor analógico y un conjunto de sensores flexibles. El principio de funcionamiento del sistema es sencillo, cada sensor es acoplado a una articulación del cuerpo, de tal forma que al doblarla sea posible medir su ángulo de flexión. El multiplexor analógico permite canalizar las señales provenientes de múltiples sensores hacia el puerto de conversión analógico/digital (A/D) del microcontrolador. Conforme las señales son digitalizadas se construye un vector, cada elemento de éste representa señal de un sensor en el momento de ser monitoreado. (Véase figura 2).

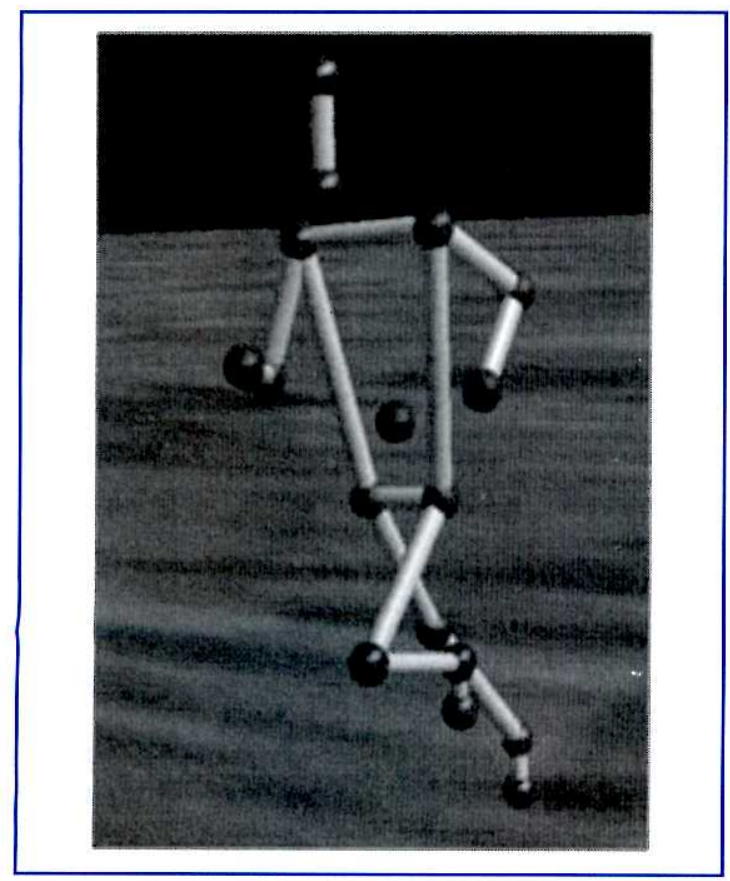

Figura 2. Modelo tridimensional del patrón de carrera humana en Visual Basic.
En su estado actual el prototipo del sistema puede monitorear las señales de hasta 64 sensores, a razón de 20 muestras por segundo por cada uno de ellos. La información obtenida puede ser enviada a una computadora a una velocidad de 9.600 bits/s, esta velocidad de transferencia es suficiente para captar aquellos movimientos que son perceptibles a simple vista.

El software de este sistema utiliza la información capturada para animar un modelo de eslabones del cuerpo humano. Esta animación muestra un movimiento suave y tiene un retardo mínimo entre la ejecución del movimiento y su representación gráfica. Otra función importante del software de este sistema es proporcionar la información capturada a otras aplicaciones a través de un servidor. (Figura 3).

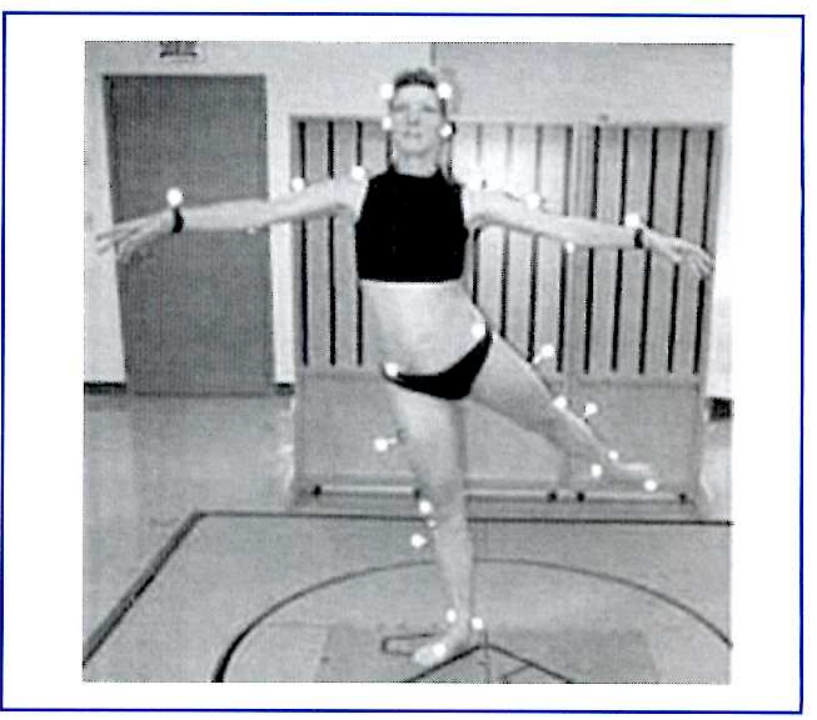

Figura 3. Captura de movimiento.

Asimismo "The motion laboratory Miami Children's Hospital" (Howell Valerie, 2000) creó un análisis del movimiento, donde se evalúa el paciente por medio de este sistema y posteriormente los resultados son procesados por una computadora, siendo aplicado a los tratamientos de individuos con alteraciones en la marcha, con el fin de adecuar las prótesis y ortesis adecuadas para cada individuo. 
Este sistema utiliza un equipo electrónico basado en elementos de computación que permiten capturar información sobre el movimiento durante la marcha y al mismo tiempo permite el registro de la actividad muscular y las fuerzas que entran en juego durante la ejecución de este movimiento. Esto proporciona un método objetivo y acertado para la evaluación y medición de la marcha con el menor riesgo de error humano e interpretación subjetiva. Este trabajo incluye el estudio de la cinemática, y la electromiografía, la cual requiere el uso de cámaras y electrodos para el registro del movimiento. La computadora procesa la información de manera sincrónica de manera que en cualquier ciclo de la marcha se conocen las posiciones de las articulaciones, la actividad muscular y la cinética del movimiento.

Por otro lado, el laboratorio de biomecánica de Blanquerna (España), creó un sistema de análisis de la marcha llamado ELICLINIC (German, Ana; Rey, Fernando; Romero, Dani, 2001) el cual es una aplicación del sistema élite para el análisis de la marcha, que consiste en un análisis biomecánico que combina tres subsistemas: análisis del movimiento, electromígrafo y una plataforma para el registro de fuerzas. Los tres sistemas trabajan sincronizadamente para permitir el estudio simultáneo del movimiento del cuerpo humano y las fuerzas que lo provocan. (Figura 4).

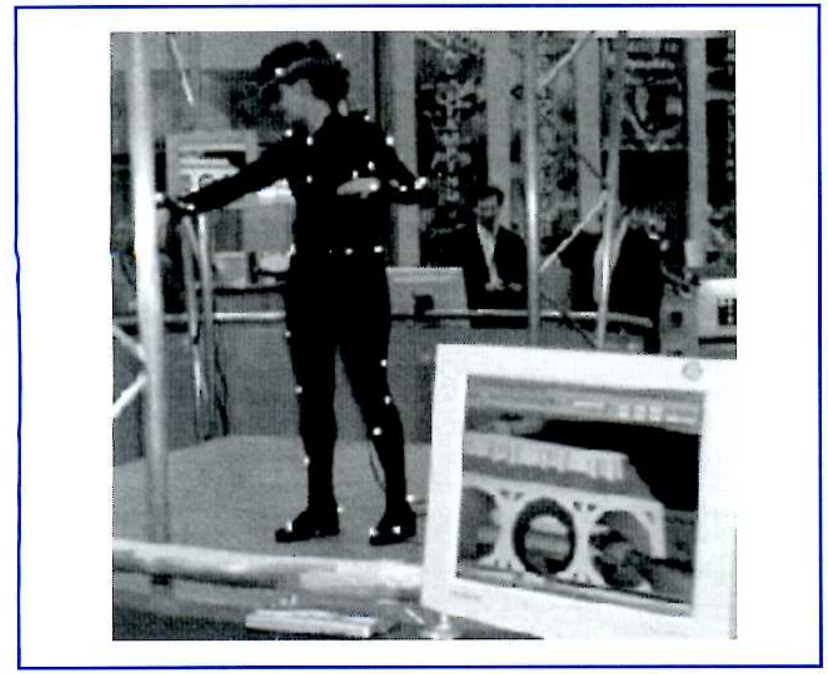

Figura 4. Simulador de movimiento.
En el Departamento de Matemáticas e Informática de la Universidad de Illes Balears (Estados Unidos), se realizó un estudio titulado Análisis y Síntesis de Movimiento Humano, donde el objetivo del trabajo fue el diseño de un sistema integrado que permitiera realizar múltiples estudios sobre el movimiento del cuerpo humano a través de un ordenador. Este sistema se basó en un modelo ya existente que permite una modelización, análisis y simulación del movimiento humano a diferentes niveles. Se obtiene a partir de una secuencia de imágenes en niveles de gris y/o color captadas desde diferentes vistas, un modelo $3 \mathrm{D}$ de la persona o personas capturadas.

Este modelo permite la animación realista de una persona, el estudio biomecánico de gestos deportivos o de baile, el reconocimiento de la persona (cara y movimientos), la integración del humanoide virtual con personajes reales, la interacción en un entorno inmersivo de persona y humanoide y el seguimiento de la persona por parte de un robot.

Este trabajo es la continuación de un sistema existente (TIC98-C302), donde se conjugan las principales técnicas actuales para realizar los procesos de análisis y síntesis del movimiento humano en un entorno común. Los sistemas tradicionales de la década de los años noventa sobre el análisis del movimiento humano aplicado (análisis del rendimiento deportivo, movimientos coreográficos, medicina, robótica) han evolucionado hacia sistemas actuales semiautomáticos basados en la implantación de marcadores ópticos que posteriormente son digitalizados a partir de imágenes estereoscópicas.

La base primordial del sistema es la utilización de un modelo biomecánico y gráfico construido a partir de una estructura jerárquica y articulada para establecer una correlación entre cada uno de los elementos estructurales del modelo (partes de la persona) con las características analíticas de las imágenes (regiones) obtenidas a partir de diferentes vistas convenientemente calibradas y sincronizadas. 
En cuanto al campo deportivo, también se han diseñado estrategias o programas con ayuda de la informática, con el objeto de realizar un adecuado análisis de movimiento, a fin de evaluar la condición del deportista frente al desarrollo de un gesto deportivo en particular.

Gracias a esto, se han generado estudios como el desarrollado por el Laboratorio de Biomecánica de Granada (Buenos Aires), donde se puede analizar la competición en deportes de equipo y conocer las características de la actividad competitiva partiendo del análisis físico (cinemático) y fisiológico de las situaciones reales de juego, para elaborar el contenido y la estructura del entrenamiento en todos los deportes (Barbero, 1998). Dentro de esta investigación se tiene en cuenta las características cinemáticas que tienen el deporte, cómo se realizan los desplazamientos, entre otros.

En Colombia, el uso de estos modelos de análisis tridimensionales aplicados para la valoración funcional se han utilizado, principalmente y con gran éxito, en el análisis de la marcha en niños con parálisis cerebral con secuelas de mielomeningocele, facilitando la decisión de un procedimiento quirúrgico correctivo y su posterior programa de rehabilitación.

En este sentido, existen esfuerzos importantes de algunas instituciones públicas y privadas relacionadas con la salud y el deporte como Coldeportes, el Centro de Análisis Biomecánico de la Universidad de Pamplona, el Instituto de Ortopedia Infantil Franklin Delano Roosevelt, la Universidad de los Andes con su grupo de investigación en biomecánica, la Universidad del Rosario con su grupo de investigación en Ejercicio Físico y la Red Nacional de Investigación en Biomecánica, por implementar el uso y el análisis de movimiento a través de este recurso tecnológico.

Hasta el momento los análisis de movimiento se han centrado en la medición de variables como la distancia recorrida, la velocidad de desplazamiento, el desplazamiento articular, la cantidad de desplazamientos que se producen, el número de aceleraciones, y la utilización del espacio por parte de los sujetos evaluados.

La versatilidad de los modelos tridimensionales pueden ser utilizados en otras áreas de desempeño por parte del fisioterapeuta como en el campo laboral, centrándose en mejorar las condiciones de trabajo y la valoración del grado de exigencia en la manipulación de cargas o los desplazamientos repetitivos dentro de la ejecución de tareas y la posibilidad de realizar diagnósticos de competencias y habilidades sensoriomotoras básicas con el fin de facilitar el desarrollo de procesos de reubicación laboral o en el diseño de puestos de trabajo. En el área de la docencia y la investigación se pueden crear actividades de capacitación, tanto en pregrado como en posgrado, relacionadas con el análisis de movimiento, la realización de proyectos de investigación dirigidos a contribuir al desarrollo tecnológico de la rehabilitación en el país como la evaluación del diseño de dispositivos creados para mejorar el patrón de marcha en pacientes discapacitados.

Otras aplicaciones de estos modelos se dan por ejemplo en los resultados posoperatorios mejorando las indicaciones y técnicas quirúrgicas. También permiten estudiar las diferentes acciones musculares que tienen actividad en cada instante de un movimiento específico, y reenseñar las actividades de la vida diaria a partir de un patrón primario. En el campo militar se pueden hacer estudios de ergonomía, diseño de maleta y calzado específicos para los terrenos de la topografía colombiana, y diseños de cómo cargar el fusil, lo que influye en el estado anímico y físico del soldado; todo esto con el fin de evitar lesiones por sobrecarga de peso y optimizar el proceso de avance de las tropas hacia su objetivo. Aunque la mayoría de los estudios tienen rangos de edad donde la marcha y postura están muy definidas, sin embargo, hay poca investigación cuando la persona la está adquiriendo (edad temprana) y cuando la está perdiendo por la vejez. Estudiar y documentar los patrones de movimiento en los niños, según su etapa de crecimiento, e 
identificar en qué etapa se van cambiando los gestos es un área importante del análisis del gesto motor en el proceso vital.

\section{Conclusiones}

Aunque el uso de modelos tridimensionales en Colombia es incipiente, es probable que en corto tiempo el uso de esta tecnología en los procesos de evaluación y diagnóstico fisioterapéutico sea muy común. Por lo tanto, es importante comenzar a indagar alrededor de la aplicación de esta tecnología que permita el desarrollo de investigación aplicada dentro de la disciplina.

Es prioritario iniciar el desarrollo de habilidades en el uso y aplicación de esta tecnología. Se hace necesario conocer y estudiar los sistemas de análisis de movimiento en informática para la comprensión del movimiento, con el fin de implementarlo y usarlo en los procesos de evaluación fisioterapéutica, permitiendo avances en el diagnóstico fisioterapéutico de la capacidad funcional dentro de la rehabilitación funcional.

Es fundamental establecer al interior de las disciplinas de la rehabilitación un lenguaje común que permita comprender el verdadero sentido de la medición científica y su importancia para la valoración de la capacidad funcional.

\section{Referencias}

Barbero Álvarez, J.C. El entrenamiento de los deportes de equipo basado en estudios biomecánicos y fisiológicos de la competición. (Octubre 1998). Departamento de Educación Física y Deportiva Universidad de Granada. Año 3, $N^{\circ} 11$, Buenos Aires.

Buades, J.M.; Perales, F.J. 2002. Human color segmentation and matching using real image sequences biomechanic model. Computer graphics and vision group. Department of computer science. EUA.

Cantani, F.; Benedetti, M.G.; Leardini, A.; Sergolini, L. 2003. Kinematic assessment of foot ankle complex. Biomechanics laboratory, Istituti ortopedici Rizzoli. Roma, Italy.
Duarte, J.; Andrade, M. 1998. Perspectiva del movimiento en la temática del análisis del gesto danzal. Monografía de la Universidad de Aragón España, introducción, págs. 2,3 www.efdeportes.com

Escamilla, R.F.; Francisco, A.C.; Fleisig, G.S.; Barrentine, S.W.; Welch, C.M.; Kayes, A.V.; SPEER, K.P.; ANDREws, J.R. A three-dimensional biomechanical analysis of sumo and conventional style deadlifts (2000). Med Sci Sports Exerc. Jul; 32(7): 1265-75. PMID: 10912892 [PubMed - indexed for MEDLINE]

German, A.; Rey, F.; Romero, D. 2001. Análisis de la marcha mediante el protocolo ELICLINC. Universitat Ramon Llull. España.

Howell, V. 2000. Análisis computarizado del movimiento. The motion laboratory Miami Children's hospital. Miami, Florida.

Mena Bejarano, B. 1998. Control postural y habilidad motriz: una reflexión desde los puntos de vista neurofisiológico y biomecánico. Ascofi, Memorias XVII Congreso Nacional de Fisioterapia, 12.

Milner N. y Quanbury C. Spastic Gait Analysis: contribution of tha Motion Lab (2003); 49 págs. 215-25 Journal New Chirul. French.

Ortega Carrillo, H.; Godoy AGUIRRE, V.; Ramos NAVA, C. Prototipo de un sistema de captura de movimiento. Dirección General de Servicios de Cómputo Académico, UNAM. México, C.P. 04510.

Perales, F.J. 2002. Human motion analysis and synthesis using computer vision and graphics techniques. State of art and applications. Computer graphics and vision group. Department of computer science.

Pezzoti, E.; Biancolini, M. 2002. Modelización CAD3 del cuerpo humano y aplicación para el análisis del movimiento. Congreso de Ingeniería. España.

Shigeru, U.; TAKeHIKo, Y. 1996. Reliability of computer based video motion analysis and electomyographic analysis of sit to stand movement. Department of physical therapy, Gunma University school of Health Sciences. Journal physical Therapy, 49-54. 